\title{
Study on Sound Absorption Performance and Transmission Behavior of Perpendicular-laid Nonwovens ${ }^{\star}$
}

\author{
Tao Yang ${ }^{\text {a,*, }}$ Xiaoman Xiong ${ }^{a}$, Rajesh Mishra ${ }^{a}$, Jan Novák ${ }^{b}$, Jiri Militky ${ }^{a}$ \\ ${ }^{a}$ Department of Materials Engineering, Faculty of Textile Engineering, Technical University of \\ Liberec, Czech Republic \\ ${ }^{\mathrm{b}}$ Department of Vehicles and Engines, Faculty of Mechanical Engineering, Technical University of \\ Liberec, Liberec 46117, Czech Republic
}

\begin{abstract}
This work deals with the study of acoustic performance of perpendicular-laid nonwovens and their relation to fabric air permeability and thermal properties. Seven perpendicular-laid nonwoven fabrics were selected to examine sound absorption like average values of sound absorption coefficients $(\bar{\alpha})$ and noise reduction coefficient (NRC) as well as air permeability and thermal insulation properties. The Brüel and Kjær impedance tube instrument, Alambeta and FX3300 Air Permeability Tester were used for evaluation of acoustic, thermal and air permeability properties, respectively. It was observed that sound absorption had insignificant correlation with thermal conductivity while it was strongly correlated with thermal resistance. And the correlation coefficient of NRC and thermal resistance was 0.9835, indicating that NRC was directly proportional to thermal resistance of perpendicular-laid nonwovens. It also was observed that $\bar{\alpha}$ was inversely proportional to air permeability, with correlation coefficient 0.95. It was concluded that a higher thermal resistance and lower air permeability suggested a better sound absorption performance for a perpendicular-laid nonwoven fabric.
\end{abstract}

Keywords: Perpendicular-laid nonwoven; sound absorption; thermal conductivity; thermal resistance; air permeability

\section{Introduction}

Nonwovens have very high porosity, high specific surface area, economical price, light weight, good elasticity and a great flexibility in air permeability, they can be presented in a large number of kinds of fiber assemblies, above characteristics decide that nonwovens have been widely applied in thermal insulation and sound absorption field [1].

Thermal properties of nonwoven fabrics are extensively studied by researchers. Generally, the thermal insulation properties of nonwoven fabric have a strong correlation with its fabric

\footnotetext{
*Project supported by project of Student Grant Competition of Technical University of Liberec for .nancial support (No. 21197).

*Corresponding author.

Email address: tao.yang@tul.cz (Tao Yang).
} 
dimensional and structural parameters [2]. Arambakam numerically investigated the thermal performance of fibrous insulation materials and concluded that heat conduction through fibrous structures increases by increasing the solid volume fraction, fiber diameter, and fibers' throughplane orientations [3]. Researchers summarized that for a fixed weight, thermal insulation increases with thickness [4]. Vallabh confirmed that fabric density is a significant factor influencing the radiation component of effective thermal conductivity and the radiative thermal conductivity decreased with increase in the fabric density [5]. Soltani and Zarrebini reported that the acoustic characteristics of woven fabrics are related to fabric structural parameters such as weave type, yarn linear density, yarn twist, and fabric thickness of woven fabric [6]. Moreover, they stated that air permeability of woven fabrics, which is strongly dependent on fabric cover factor, can be used as a criterion of sound absorption behavior. Yang and Yu conducted experimental study and found that nonwoven fabrics with highest value of air permeability exhibit inferior acoustic absorbency [7]. Kucuk M and Korkmaz Y measured sound absorption properties of eight different nonwoven composites including different fiber types mixed with different ratios, they stated that the increase in thickness and the decrease in air permeability results in an increase in sound absorption properties of the material [8]. Perpendicular-laid nonwovens exhibit excellent compression recovery, softness and very good sound absorption performance since perpendicular-laid nonwovens have highloft structure where all the fibers are orientated in the vertical plane $[9,10,11]$. Tascan and Vaughn investigated the acoustical insulation of perpendicular-laid nonwoven fabrics made from different polyester fibers, and stated that fabrics made from 3 denier fibers have better sound insulation than those made from 15 denier fibers. It was also found that perpendicular-laid nonwoven fabrics made from 4DG and trilobal fibers showed better sound insulation than those made from round fibers $[12,13]$. Klara Kalinova reported that perpendicular-laid nonwovens (perpendicularly laid fiber web) have better sound absorption performance than longitudinally laid fiber web [14].

Since the transmission behavior and acoustic property of nonwoven both strongly depend on its structure parameters, for a specified nonwoven fabric there should be some relation between these performances. However, there is very limited research devoted to understanding this relationship. In this paper, seven perpendicular-laid nonwovens were selected to measure sound absorption properties, air permeability and thermal performance. The relationship between sound absorption and thermal properties were studied as well.

\section{Methodology}

\section{$2.1 \quad$ Materials}

Seven perpendicular-laid nonwoven fabrics made by Technical University of Liberec were selected to measure transmission and acoustic properties. The vibrating perpendicular lapper used to fabricate perpendicular-laid nonwovens is illustrated in Fig. 1. The vibrating perpendicular lapper consists of a carding machine, a perpendicular-laid vertical lapper and a through-air thermobonding chamber [13]. The carded web is fed onto conveyor belt and a reciprocating forming comb pulls the carded web towards the hold back roller to form a fold. The fold is pulled off the comb by a system of needles placed on a reciprocating compressing bar and pushed to the fiber layer which is created and moved between the conveyor belt and a wire grid. The fiber layer is bonded by melting bonding fibers present in the fiber blend when it passes through thermo-bonding cham- 


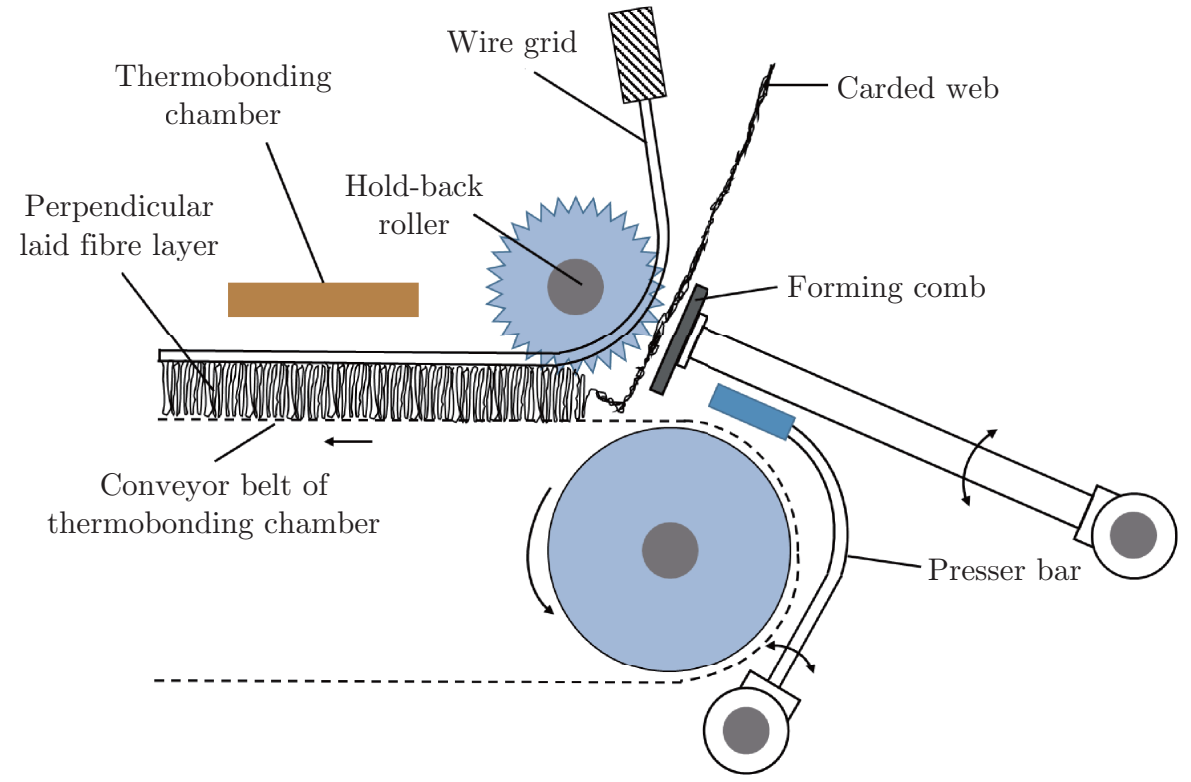

Fig. 1: Vibrating perpendicular lapper

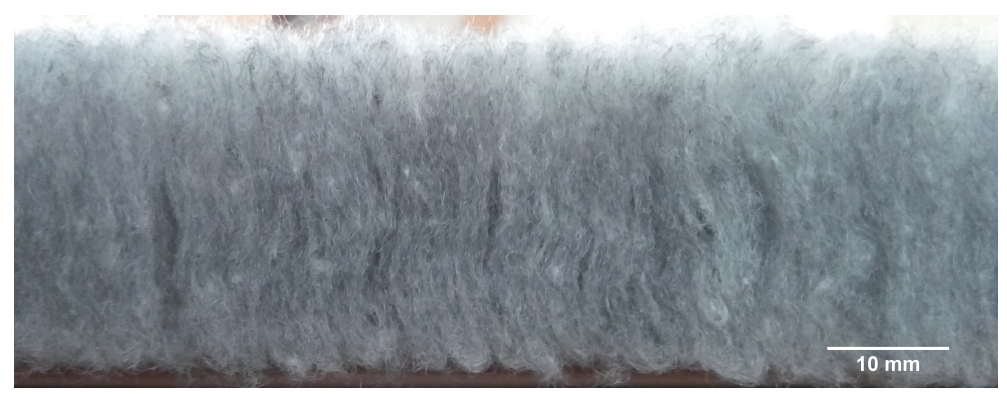

Fig. 2: Structure of perpendicular-laid nonwoven

ber [15]. In Perpendicular-laid nonwoven structure, fibers are orientated in the vertical plane as shown in Fig. 2, this gives the highest possible resilience and recovery after repeated compression.

The perpendicular-laid nonwoven samples were made by different types of polyester fibers, the fiber specifications are shown in Table 1. The characteristics of the specimens are shown in Table 2. Fabric thickness were measured by Alambeta. Fabric mass per unit area were determined according to ISO 9073-1:1989. Perpendicular-laid nonwovens' porosity values were determined according to ASTM C 830-00 Standard Test Methods for Apparent Porosity, Liquid Absorption, Apparent Specific Gravity, and Bulk Density of Refractory Shapes by Vacuum Pressure using following equation [16]:

$$
h=\left(1-\frac{\rho_{w}}{\rho_{f}}\right) \times 100 \%
$$

where $h$ is porosity, $\rho_{w}$ is fabric density, and $\rho_{f}$ is weighted average absolute density of the fibers. For hollow fibers, as only the pores that carry air flow in connection with the ambient air are of interest in terms of sound absorption, the voids in hollow fibers are not included in the porosity $[17,18,19]$. 
Table 1: Fiber specifications

\begin{tabular}{clcccc}
\hline $\begin{array}{c}\text { Fiber } \\
\text { Code }\end{array}$ & Types of PET & $\begin{array}{c}\text { Diameter } \\
(\mu \mathrm{m})\end{array}$ & $\begin{array}{c}\text { Fineness } \\
(\mathrm{dtex})\end{array}$ & $\begin{array}{c}\text { Staple Length } \\
(\mathrm{mm})\end{array}$ & $\begin{array}{c}\text { Ratio of Core } \\
\text { and Sheath }\end{array}$ \\
\hline i & Hollow PET & 28.64 & 4.92 & 70.00 & - \\
ii & PET & 15.25 & 1.76 & 50.00 & - \\
iii & Bicomponent PET & 22.02 & 3.24 & 50.00 & $3: 1$ \\
iv & PET & 26.91 & 6.70 & 57.00 & - \\
v & Bicomponent PET & 14.58 & 2.20 & 38.00 & $3: 1$ \\
\hline
\end{tabular}

Table 2: Characteristics of perpendicular-laid nonwovens

\begin{tabular}{cccccc}
\hline $\begin{array}{c}\text { Samples } \\
\text { code }\end{array}$ & Content & $\begin{array}{c}\text { GSM } \\
\left(\mathrm{g} / \mathrm{m}^{2}\right)\end{array}$ & $\begin{array}{c}\text { Thickness } \\
(\mathrm{mm})\end{array}$ & $\begin{array}{c}\text { Porosity } \\
(\%)\end{array}$ & $\begin{array}{c}\text { Bulk density } \\
\left(\mathrm{kg} / \mathrm{m}^{3}\right)\end{array}$ \\
\hline A & $30 \%$-i & 507.51 & 24.09 & 97.57 & 20.07 \\
B & $45 \%$-ii & 478.32 & 28.36 & 98.06 & 16.87 \\
C & $25 \%$-iii & 465.22 & 27.48 & 98.05 & 16.93 \\
D & & 335.68 & 20.82 & 98.67 & 16.12 \\
E & $70 \%$-iv & 317.51 & 19.85 & 98.69 & 15.99 \\
F & $30 \%-\mathrm{v}$ & 198.64 & 20.12 & 99.19 & 9.87 \\
G & & 259.28 & 20.66 & 98.97 & 12.55 \\
\hline
\end{tabular}

\subsection{Methods}

\subsubsection{Evaluation of Sound Absorption Performance}

Sound absorption of perpendicular-laid nonwovens were measured according to ASTM E 1050: Standard Test Method for Impedance and Absorption of Acoustical Properties Using a Tube, Two Microphones and a Digital Frequency Analysis System, which was developed to determine the ability of materials for absorbing normal incidence sound waves. A Brüel and Kjær measuring instrument containing Type 4206 Impedance Tube, PULSE Analyzer Type 3560, and Type 7758 Material Test Software was used for testing within the frequency range $50 \mathrm{~Hz}-6.4 \mathrm{kHz}$. A large tube (100 $\mathrm{mm}$ in diameter) and a small tube (29 $\mathrm{mm}$ in diameter) were set up for measuring the sound absorption in low-frequency range from $50-1600 \mathrm{~Hz}$ and high-frequency range 500-6400 Hz respectively. The curves from both measurements were merged. The sound absorption values of lower common frequencies (start from 50 to $1600 \mathrm{~Hz}$ ) were mainly from the large tube and the higher common frequencies (up to $1600 \mathrm{~Hz}$ ) were mainly from the small tube. The testing principle is illustrated in Fig. 3.

A sound source is mounted at one end of the impedance tube and the material sample is placed at the other end. The loudspeaker generates broadband, stationary and random sound waves. These incident sound signals propagate as plane waves in the tube and hit the sample surface. The reflected wave signals are picked up and compared to the incident sound wave. Sound absorption of five different samples from each material was measured. 


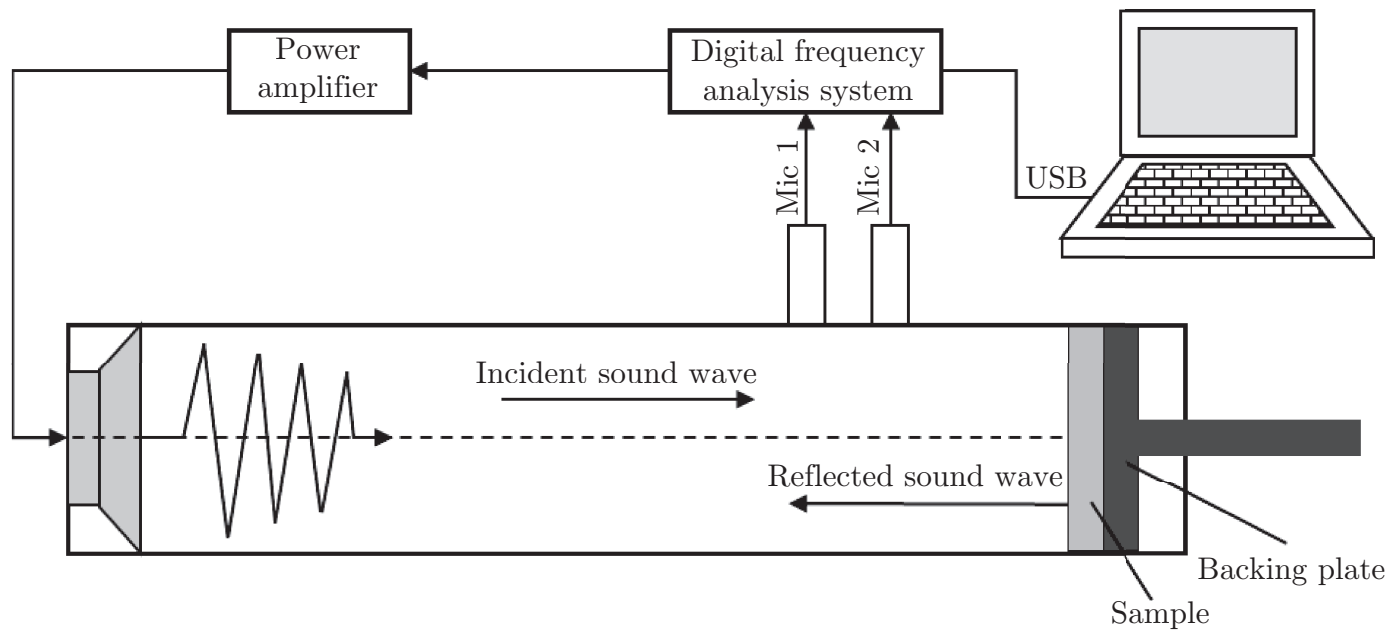

Fig. 3: Measuring system configuration

\subsubsection{Measurement of Air Permeability}

Air permeability measures the ability of a porous medium to transmit fluids. It depends on the porous geometrical structure. The air permeability of perpendicular-laid nonwovens was measured using FX3300 Textech Air Permeability Tester Fig. 4. The fabric sample is fixed as an obstacle in a flow of air by the clamping holder. A pressure difference $\Delta p$ between both sides of the fabric sample develops as a consequence of hydraulic losses. The pressure difference is recorded by using of the manometer. The measured value is a speed of air in meter per second or a volume rate of the flow in liter per hour.

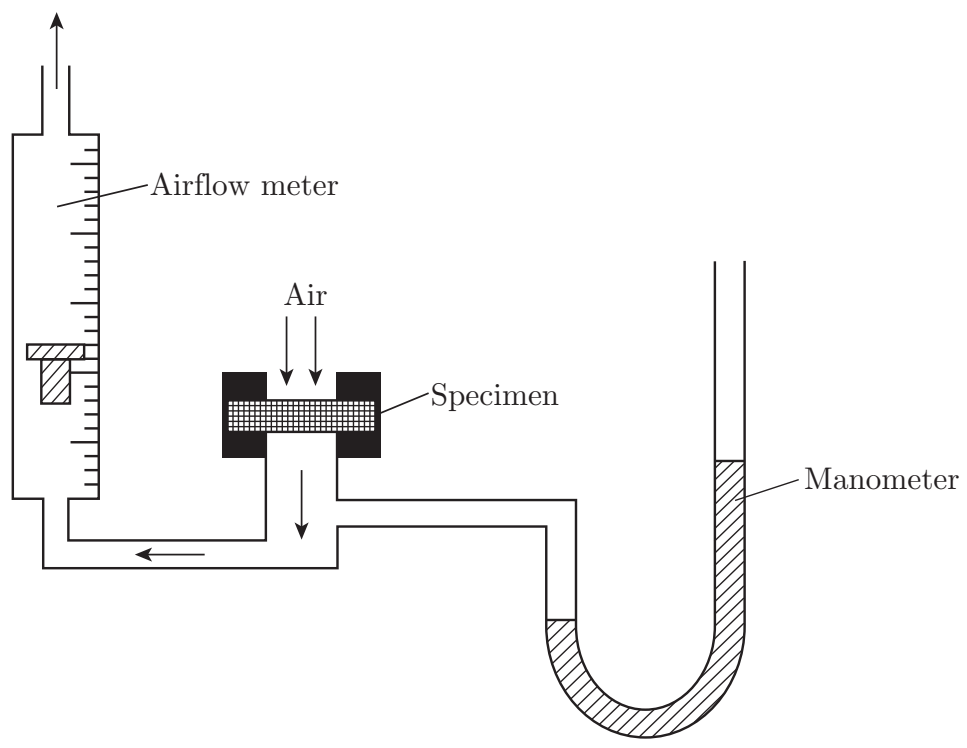

Fig. 4: Set-up for measuring air permeability

\subsubsection{Measurement of Thermal Properties}

Alambeta instrument was used to measure thermal conductivity and thermal resistance, according to EN 31092 standard. The measuring head of the Alambeta contains a copper block which 
is electrically heated to approximately $32^{\circ} \mathrm{C}$ to simulate human skin temperature, which is maintained by a thermometer connected to the regulator. The lower part of the heated block is equipped with a direct heat flow sensor which measures the thermal drop between the surfaces of a very thin, non-metallic plate using a multiple differential micro-thermocouple [20]. Each specimen was tested five times and the results were averaged.

\section{Thermal conductivity}

Thermal conductivity, $\lambda$, measures the rate at which heat is transferred through unit area of the fabric across unit thickness under a specified temperature gradient and thus is defined by the relation $[21]$

$$
\lambda\left(W \cdot m^{-1} \cdot K^{-1}\right)=\frac{Q}{F \tau \frac{\Delta T}{h}}
$$

where $Q$ is the amount of conducted heat, $F$ is the area through which heat is conducted, $\tau$ is the time of heat conduction, $\Delta T$ is the gradient of the temperature and $h$ is the fabric thickness.

\section{Thermal resistance}

Thermal resistance expresses the ability of material to prevent heat flow through the thickness over unit surface area. Fabric thickness and thermal conductivity are important factors governing thermal insulation of textiles. Usually, the higher the thermal resistance, the lower the heat loss. The thermal resistance, $R$, is connected with the thermal conductivity, $\lambda$, and the fabric thickness, $h$, as follows

$$
R\left(m^{2} \cdot K \cdot W^{-1}\right)=\frac{h}{\lambda}
$$

\section{Results and Discussion}

\subsection{Sound Absorption Coefficient}

Sound absorption coefficient indicates how much of the sound is absorbed by the testing material. The absorption coefficient can be expressed as

$$
\alpha=\frac{I_{a}}{I_{i}}
$$

where $I_{a}$ is sound intensity absorbed in $\mathrm{W} / \mathrm{m}^{2}$ and $I_{i}$ is incident sound intensity in $\mathrm{W} / \mathrm{m}^{2}$.

The normal incidence sound absorption coefficient $(\alpha)$ of perpendicular-laid nonwovens were determined as a function of the sound frequency $(f)$, as shown in Fig. 5. The measured data in the low frequency range of 50-1600 Hz (using the Type 4206 large tube) and the high frequency range (500-6400 Hz tested by the Type 4206 small tube) were combined together to form plotted curves.

It is observed that sound absorption coefficient of the test samples lies in the range of 0.0170.76. Apparently, the value of absorption coefficient for samples A, B and C sharply increases at frequency bands $50 \mathrm{~Hz}-3500 \mathrm{~Hz}$ and the maximum value of absorption coefficient occurs at frequency bands $4000 \mathrm{~Hz}-5400 \mathrm{~Hz}$. However, samples D, E, F and G show lower absorption coefficient value in comparison to samples $\mathrm{A}, \mathrm{B}$ and $\mathrm{C}$, and the value of absorption coefficient 


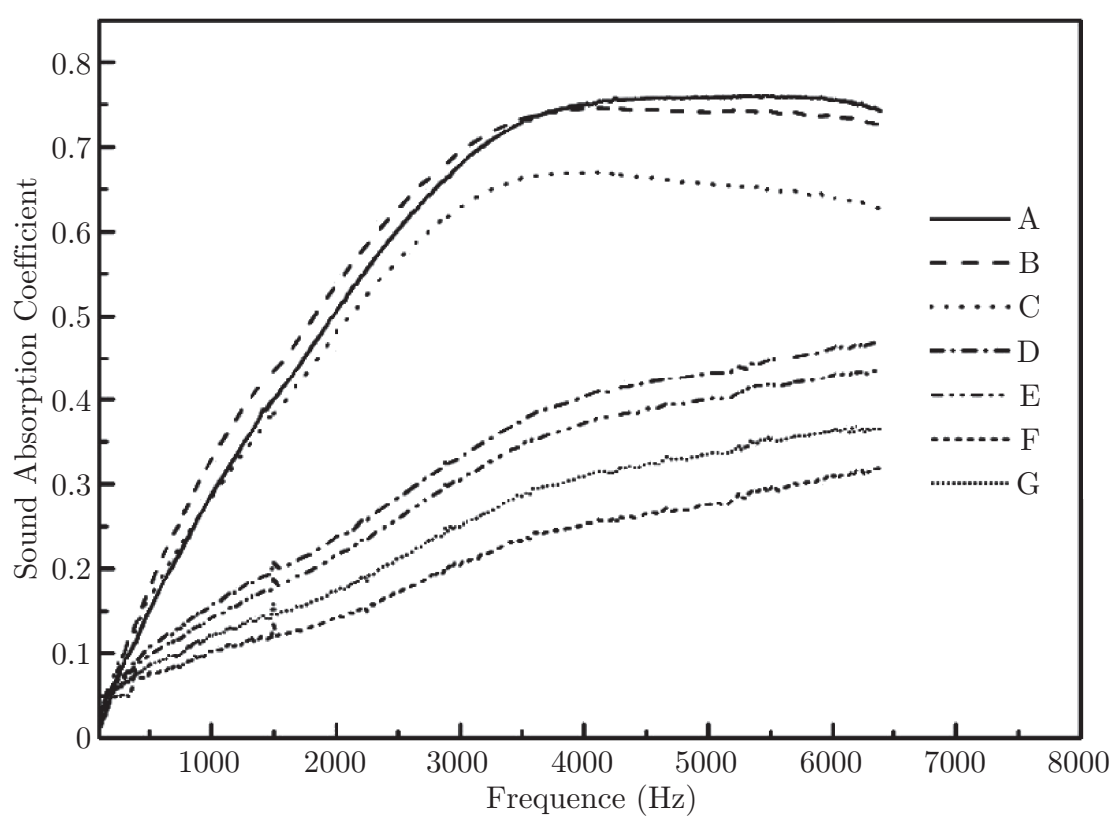

Fig. 5: Sound absorption coefficient of perpendicular-laid nonwovens

increases with the increasing of frequency at the whole measurable frequency bands (50 Hz-6400 $\mathrm{Hz}$ ). Results indicate that perpendicular-laid nonwoven exhibits much better sound absorption ability at frequency bands $3000 \mathrm{~Hz}-6400 \mathrm{~Hz}$.

For a numerical comparison, the noise reduction coefficient (NRC) and average values of sound absorption coefficients $(\bar{\alpha})$ for all the nonwovens were calculated. The NRC is the average value of Sabin alphas for 250, 500, 1000 and $2000 \mathrm{~Hz}$, which provides a decent and simple quantification of how well the porous material will absorb the human voice. Average value of sound absorption coefficient $(\bar{\alpha})$ is the average of the absorption coefficients for a specific frequency bands. The higher the $\bar{\alpha}$, the better the material absorbs sound. The $\bar{\alpha}$ of perpendicular-laid nonwovens is calculated using the following equation:

$$
\begin{gathered}
N R C=\frac{\alpha_{250 \mathrm{~Hz}}+\alpha_{500 \mathrm{~Hz}}+\alpha_{1000 \mathrm{~Hz}}+\alpha_{2000 \mathrm{~Hz}}}{4} \\
\bar{\alpha}=\frac{\int_{F_{1}}^{F_{2}} \alpha(f) d f}{F_{2}-F_{1}}
\end{gathered}
$$

where $f$ is sound frequency, $F_{1}(50 \mathrm{~Hz})$ is lower bound of sound frequency in testing and $F_{2}(6400$ $\mathrm{Hz}$ ) is upper bound of sound frequency in measuremen.

The computed NRC and $\bar{\alpha}$ values between $50 \mathrm{~Hz}$ and $6400 \mathrm{~Hz}$ for perpendicular-laid nonwovens are shown in Table 3. The NRC value of seven perpendicular-laid nonwovens is found to lie in the range of 0.092-0.281, indicating perpendicualr-laid nonwovens are not effective absorber for low-frequency sound absorption. It is found that the $\bar{\alpha}$ values of perpendicular-laid nonwovens are ranging from 0.201 to 0.588 . It is also observed that $\mathrm{A}, \mathrm{B}$ and $\mathrm{C}$ exhibit higher ability for sound absorption, superior to D, E, F and G as shown in Fig. 4 and Table 3. This can be explained by their differences in fabric thickness, GSM and fiber fineness. Fabric thickness is a very important factor determining the sound absorption ability of nonwoven structures. The thickness of sound absorber has direct relationship with sound absorption ability. As the material gets thicker, the sound absorption at low frequencies increases as well $[22,23]$. Generally, vibrations of finer fibers 
are easier compared to coarser fibers on incidence of sound waves, and therefore the content of finer fibers increases sound absorption coefficient values due to an increase in airflow resistance by means of friction of viscosity through the vibration of the air [24, 25, 26]. Thus, the smaller fiber diameters in samples $\mathrm{A}, \mathrm{B}$ and $\mathrm{C}$ could be one reason accounting for their better sound absorption performance. For high GSM fabrics (samples A, B and C) there are more fibers involved in the viscous losses and more acoustic energy is dissipated in the form of heat energy [27].

Table 3: NRC and mean of SAC $(\bar{\alpha})$

\begin{tabular}{cccccccc}
\hline \multirow{2}{*}{ Sample codes } & \multicolumn{3}{c}{ NRC } & & \multicolumn{3}{c}{ SAC $(\bar{\alpha})$} \\
\cline { 2 - 3 } \cline { 7 - 8 } & Mean value & SD & CV & & Mean value & SD & CV \\
\hline A & 0.254 & 0.003 & 0.013 & & 0.580 & 0.008 & 0.015 \\
B & 0.281 & 0.003 & 0.009 & & 0.588 & 0.007 & 0.012 \\
C & 0.255 & 0.005 & 0.020 & & 0.524 & 0.006 & 0.011 \\
D & 0.142 & 0.010 & 0.069 & & 0.315 & 0.028 & 0.089 \\
E & 0.129 & 0.006 & 0.047 & & 0.291 & 0.017 & 0.060 \\
F & 0.092 & 0.004 & 0.046 & & 0.201 & 0.007 & 0.035 \\
G & 0.110 & 0.005 & 0.043 & & 0.243 & 0.014 & 0.056 \\
\hline
\end{tabular}

\subsection{Air Permeability}

In order to examine the effect of fiber deformation on the measured air permeability of perpendicularlaid nonwovens, different pressure gradients $50 \mathrm{~Pa}, 100 \mathrm{~Pa}, 150 \mathrm{~Pa}$ and $200 \mathrm{~Pa}$ were chosen to carry out air permeability testing. The measured air permeability is listed in Table 4 . Each value is the average of five test results.

Since perpendicular-laid nonwoven is loose fabric, the large spacing between fibers enables the majority of air to flow through these gaps. Obviously, samples A, B and $\mathrm{C}$ exhibit much lower air permeability while $\mathrm{F}$ and $\mathrm{G}$ show the highest air permeability. Similar to the sound absorption performance, this is also attributed to their difference in GSM and fabric thickness. For perpendicular-laid nonwovens with higher GSM and fabric thickness, there is less pores and air space in textile structure to allow air flow go through.

\subsection{Thermal Properties}

Thermal conductivity and thermal resistance of perpendicular-laid nonwovens are presented in Table 5. For nonwovens with approximately the same thickness (samples D, E, F and G), thermal resistance increases with the increasing of fabric areal density and thermal conductivity shows an adverse trend. This is because increase in GSM causes increase in fiber to fiber contact and packing density as well as tortuosity, so less heat flows through the channels in nonwoven, and thermal resistance therefore increases correspondingly. Moreover, for samples A, B and C, since they have slightly different GSM and varying thickness, the increase of thermal resistance with the increase in sample thickness indicated that fabric thickness plays a major role in deciding thermal resistance of perpendicular-laid nonwovens. 
Table 4: Measured air permeability of samples

\begin{tabular}{ccccc}
\hline \multirow{2}{*}{ Samples Code } & \multicolumn{4}{c}{ Air Permeability $(\mathrm{mm} / \mathrm{s})$} \\
\cline { 2 - 5 } & $\Delta \mathrm{P}=50 \mathrm{~Pa}$ & $\Delta \mathrm{P}=100 \mathrm{~Pa}$ & $\Delta \mathrm{P}=150 \mathrm{~Pa}$ & $\Delta \mathrm{P}=200 \mathrm{~Pa}$ \\
\hline $\mathrm{A}$ & $320.1 \pm 18.4177$ & $597.6 \pm 33.781$ & $848.7 \pm 47.8773$ & $1070.5 \pm 63.1774$ \\
$\mathrm{~B}$ & $320.1 \pm 58.586$ & $652.1 \pm 74.994$ & $923.3 \pm 102.373$ & $1177.6 \pm 136.177$ \\
$\mathrm{C}$ & $370.6 \pm 15.4071$ & $687.3 \pm 27.713$ & $968.3 \pm 36.0032$ & $1227 \pm 46.4399$ \\
$\mathrm{D}$ & $908.8 \pm 33.4171$ & $1598 \pm 58.0517$ & $2166 \pm 74.027$ & $2712 \pm 95.2365$ \\
$\mathrm{E}$ & $929.8 \pm 59.6674$ & $1628 \pm 96.2808$ & $2220 \pm 130.192$ & $2760 \pm 159.844$ \\
$\mathrm{~F}$ & $1548 \pm 42.0714$ & $2608 \pm 95.2365$ & $3508 \pm 167.093$ & $4336 \pm 308.432$ \\
$\mathrm{G}$ & $1234 \pm 30.4959$ & $2104 \pm 42.7785$ & $2824 \pm 58.9915$ & $3468 \pm 40.8656$ \\
\hline
\end{tabular}

Note: Samples were held by clamping arm under the load $50 \mathrm{~N}$

Table 5: Thermal properties of perpendicular-laid nonwovens

\begin{tabular}{|c|c|c|c|c|c|c|}
\hline \multirow{2}{*}{ Sample codes } & \multicolumn{3}{|c|}{ Thermal conductivity } & \multicolumn{3}{|c|}{ Thermal resistance } \\
\hline & $\begin{array}{c}\text { Mean value } \\
10^{-3} \mathrm{~W} \cdot \mathrm{m}^{-1} \cdot \mathrm{K}^{-1}\end{array}$ & $\mathrm{SD}$ & $\mathrm{CV}$ & $\begin{array}{c}\text { Mean value } \\
10^{-3} \mathrm{~K} \cdot \mathrm{m}^{2} \cdot \mathrm{W}^{-1}\end{array}$ & SD & $\mathrm{CV}$ \\
\hline A & 55.84 & 1.86 & 0.03 & 431.80 & 15.48 & 0.04 \\
\hline B & 61.70 & 3.28 & 0.05 & 460.60 & 22.78 & 0.05 \\
\hline $\mathrm{C}$ & 60.04 & 1.95 & 0.03 & 458.20 & 18.71 & 0.04 \\
\hline $\mathrm{D}$ & 63.30 & 1.35 & 0.02 & 329.20 & 6.61 & 0.02 \\
\hline $\mathrm{E}$ & 63.42 & 0.59 & 0.01 & 313.00 & 13.17 & 0.04 \\
\hline F & 71.10 & 0.70 & 0.01 & 283.00 & 6.36 & 0.02 \\
\hline G & 67.00 & 1.49 & 0.02 & 308.40 & 16.04 & 0.05 \\
\hline
\end{tabular}

\subsection{Relation between Sound Absorption Performance and Air Per- meability}

Estimated correlation between air permeability and sound absorption is presented in Fig. 6. It is observed that $\bar{\alpha}$ has significant correlation with air permeability, the correlation coefficient is about 0.95, indicating the existence of an inverse relation between air permeability and sound absorption of perpendicular-laid nonwovens. That means perpendicular-laid nonwoven with lower air permeability usually exhibits better sound absorption performance. This may provide a new method to evaluate the sound absorption property of perpendicular-laid nonwovens by means of air permeability testing [28].

\subsection{The Relationship between Acoustic and Thermal Properties}

Fig. 7 illustrates the estimation of correlation between thermal conductivity of perpendicular-laid nonwovens and sound absorption, NRC and average value of SAC $(\bar{\alpha})$. It is observed that both NRC and $\bar{\alpha}$ have insignificant correlation with thermal conductivity, the correlation coefficients are 0.60353 and 0.68973 , respectively. The reason could be that thermal conductivity is not so 


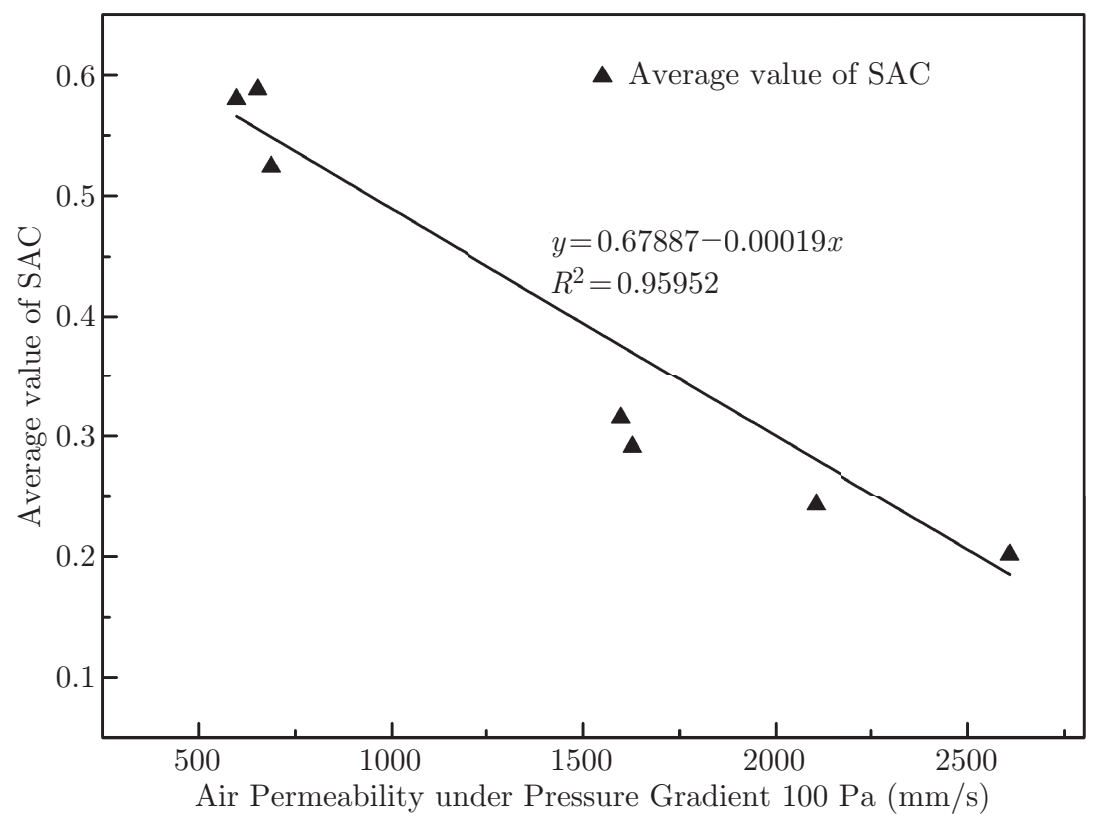

Fig. 6: Estimated correlation between air permeability and sound absorption

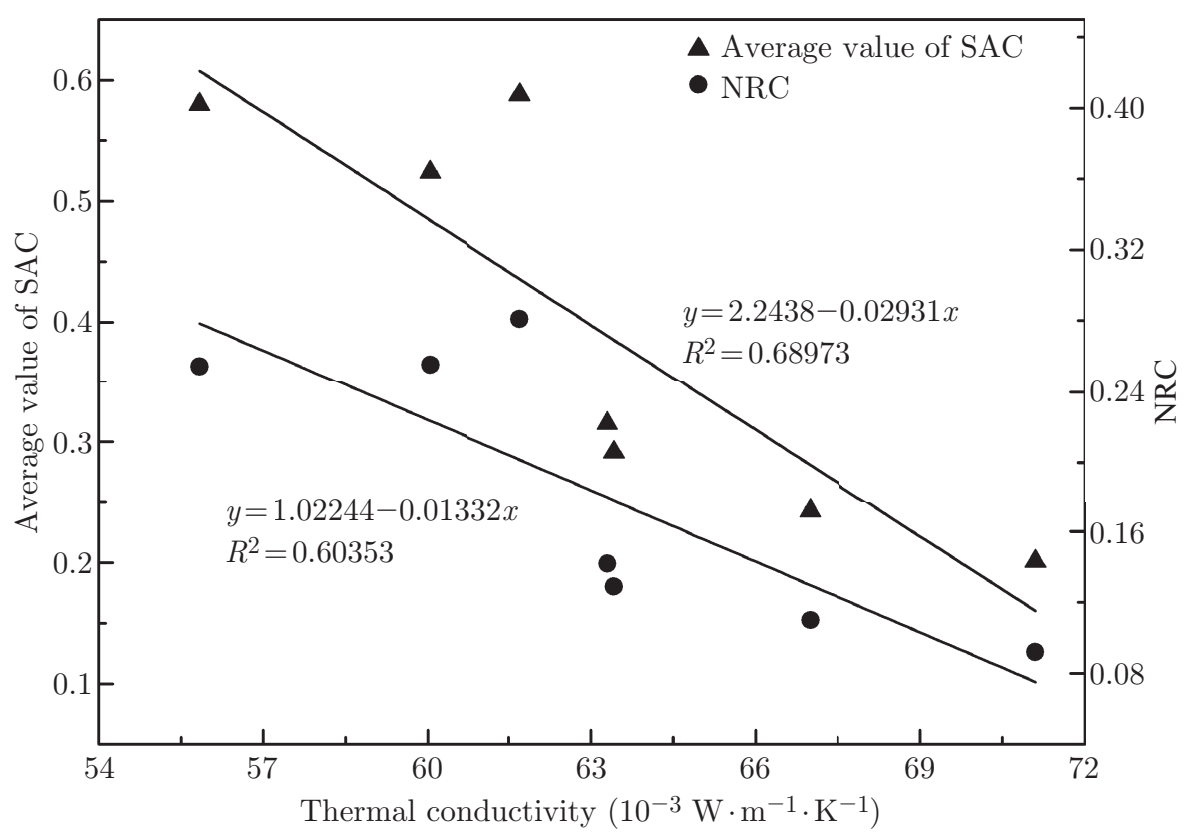

Fig. 7: Estimation of correlation between thermal conductivity and sound absorption

strongly dependent on fabric thickness while fabric thickness is a determining factor for sound absorption.

Estimation of correlation between thermal resistance and sound absorption, NRC and $\bar{\alpha}$, are presented in Fig. 8. The correlation coefficient between NRC and thermal resistance is 0.9835, and this value is 0.94853 for $\bar{\alpha}$ and thermal resistance, indicating that the sound absorption performance of perpendicular nonwovens has a very strong correlation with thermal resistance. It can be concluded that NRC and $\bar{\alpha}$ are directly proportional to thermal resistance of perpendicular nonwovens. That means for different perpendicular nonwovens, a higher thermal resistance 


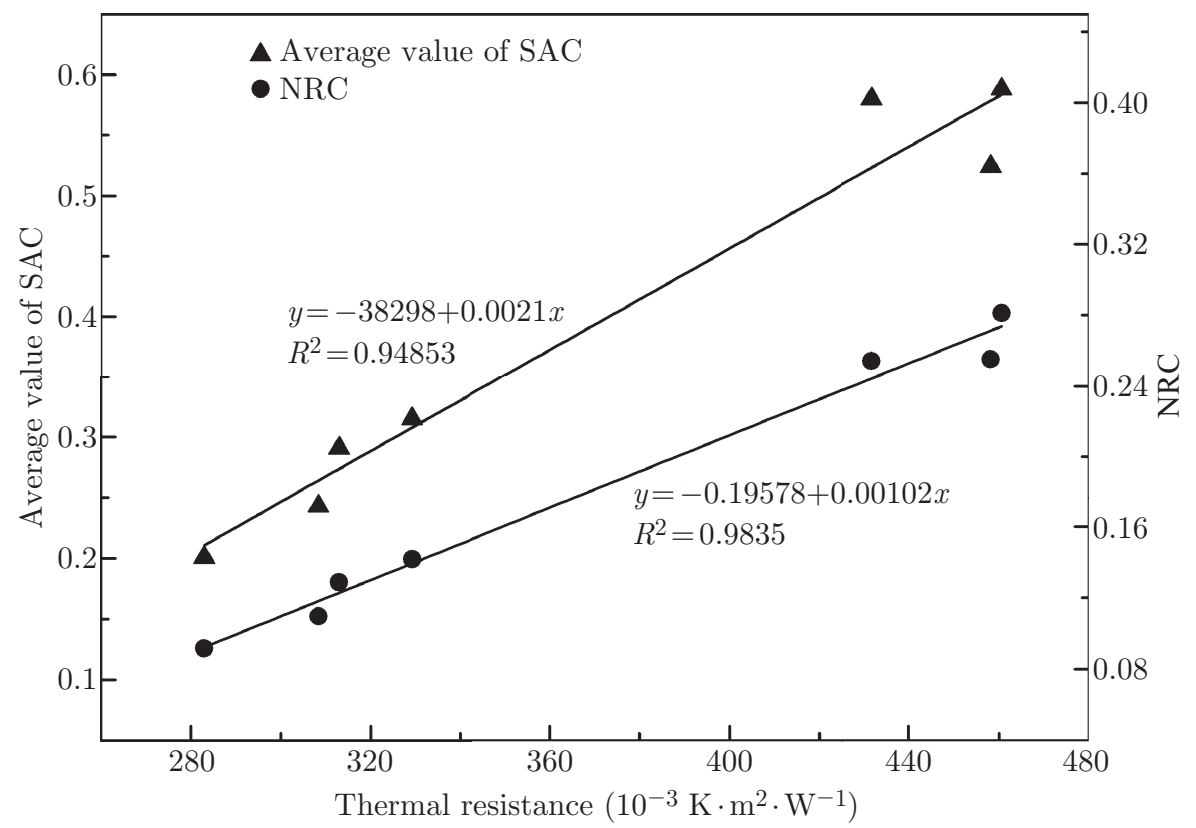

Fig. 8: Estimation of correlation between thermal resistance and sound absorption

suggests a better sound absorption performance [29].

\section{Conclusion}

Seven perpendicular-laid nonwovens were selected to investigate their acoustic property as well as air permeability and thermal properties. Results showed that perpendicular-laid nonwoven is ineffective for sound absorption at frequency lower than $3000 \mathrm{~Hz}$ while it exhibits much better sound absorption ability at frequency bands $3000 \mathrm{~Hz}-6400 \mathrm{~Hz}$. Results indicated that there exists an inverse relation between air permeability and sound absorption of perpendicular-laid nonwovens. It can be stated that a lower air permeability suggests a better sound absorption performance for a perpendicular-laid nonwoven fabric. It is also observed that sound absorption has insignificant correlation with thermal conductivity while it is strongly correlated with thermal resistance. The correlation coefficient NRC and thermal resistance is 0.9835 , which indicates that NRC is directly proportional to thermal resistance of perpendicular-laid nonwovens. Thus, a higher thermal resistance suggests a better sound absorption performance for a perpendicularlaid nonwoven fabric.

\section{Acknowledge}

This work was supported by the research project of Student Grant Competition of Technical University of Liberec No. 21197 granted by Ministry of Education Youth and Sports of Czech Republic. 


\section{References}

[1] Thilagavathi G, Pradeep E, Kannaian T, Sasikala L. Development of Natural Fiber Nonwovens for Application as Car Interiors for Noise Control. J IND TEXT: 2010; 39: 267-278.

[2] Xiong X, Yang T, Huang J, et al. Heat transfer through thermal insulation materials Part INonwoven fabrics. Recent developments in fibrous material science 2015; 2: 85-104.

[3] Arambakam R, Vahedi Tafreshi H and Pourdeyhimi B. A simple simulation method for designing fibrous insulation materials. Mater Design: 2013; 44: 99-106.

[4] Behera BK, Mishra R. Artificial neural network-based prediction of aesthetic and functional properties of worsted suiting fabrics. Int J Cloth Sci Tech: 2007; 19: 259-276.

[5] Vallabh R, Banks-Lee P and Mohammadi M. Determination of radiative thermal conductivity in needle punched nonwovens. J Eng Fiber Fabr: 2008; 3: 46-52.

[6] Soltani P and Zarrebini M. Acoustic performance of woven fabrics in relation to structural parameters and air permeability. J Text I: 2013; 104(9): 1011-1016.

[7] Yang $\mathrm{S}$ and $\mathrm{Yu} \mathrm{W}$. Air permeability and acoustic absorbing behaviour of nonwovens. J Fiber Bioeng Infor: 2011; 3: 203-207.

[8] Kucuk M and Korkmaz Y. The effect of physical parameters on sound absorption properties of natural fiber mixed nonwoven composites. Text Res J: 2012, 82(20): 2043-2053.

[9] Vasile S and Van-Langenhove L. Automotive industry a high potential market for nonwovens sound insulation. J Text Appl Tech Mgmt: 2004; 3: 1-5.

[10] Parikh DV, Calamari TA, Goynes WR, Chen Y and Jirsak O. Compressibility of cotton blend perpendicular-laid nonwovens. Text Res J: 2004; 74: 7-12.

[11] Jirsak O, Burian T and Sasková P. Improvements in Compressional Properties of Highlofts. Fibred Text East Eur: 2003; 11(3): 80-83.

[12] Tascan M and Vaughn EA. Effects of fiber denier, fiber cross-sectional shape and fabric density on acoustical behavior of vertically lapped nonwoven fabrics. J Eng Fiber Fabr: 2008; 3(2): 32-38.

[13] Tascan $M$ and Vaughn EA. Effects of total surface area and fabric density on the acoustical behavior of needle punched nonwoven fabrics. Text Res J: 2008; 78(4): 289-296.

[14] Kalinova K. Theoretical assessment of sound absorption coefficient for anisotropic nonwovens. QScience Connect: 2013; 3: 3-10.

[15] Jirsak O and Wadsworth L. Nonwoven Textiles. Durham, NC: Carolina Academic Pr. 1998: 57-58.

[16] American Society for Testing and Materials. ASTM C 830-00 standard test methods for apparent porosity, liquid absorption, apparent specific gravity, and bulk density of refractory shapes by vacuum pressure. ASTM: Westcohocken, PA, 2000.

[17] Yilmaz ND, Powell NB, Banks-Lee P and Michielsen S. Multi-fiber needle-punched nonwoven composites: Effects of heat treatment on sound absorption performance. J Ind Text: 2013, 43(2), 231-246.

[18] McRae JD, Naguib HE and Atalla N. Mechanical and acoustic performance of compression-molded open-cell polypropylene foams. J Appl Polym Sci: 2010; 116(2): 1106-1115.

[19] Yilmaz ND, Michielsen S, Banks-Lee P, et al. Effects of material and treatment parameters on noise control performance of compressed three-layered multifiber needlepunched nonwovens. J Appl Polym Sci: 2012; 123(4): 2095-2106.

[20] Hes L and Dolezal I. New method and equipment for measuring thermal properties of textiles. Journal of the Textile Machinery Society of Japan: 1989; 42: 124-128.

[21] Gun AD. Dimensional, physical and thermal comfort properties of plain knitted fabrics made from modal viscose yarns having microfibers and conventional fibers. Fiber Polym: 2011; 12: 258-267. 
[22] Coates M and Kierzkowski M. Acoustic textiles: lighter, thinner and more sound absorbent. Tech Text Int: 2002; 11(7), 15-18.

[23] Ibrahim MA and Melik RW, Physical Parameters Affecting Acoustic Absorption Characteristics of Fibrous Materials, Proceedings of the mathematical and physical society of Egypt: 1978, 46.

[24] Seddeq HS. Factors influencing acoustic performance of sound absorptive materials. Australian Journal of Basic and Applied Sciences: 2009; 3: 4610-4617.

[25] Lee Y and Joo C. Sound absorption properties of recycled polyester fibrous assembly absorbers. AUTEX Res J: 2003, 3(2), 78-84.

[26] Sun F, Banks-Lee P and Peng H. Sound absorption in an anisotropic periodically layered fluidsaturated porous medium. Appl Acoust: 1993, 39(1-2), 65-76.

[27] Yang T, Xiong X, Mishra R, Novak J and Militky J. Acoustic evaluation of Struto nonwovens and their relationship with thermal properties. Textile Research Journal: 2016; 4051751668195.

[28] Yang T, Xiong X, Mishra R, Novák J, Chaloupek J, Sanetrnik F and Militký J. Investigation on acoustic behavior and air permeability of struto nonwovens. Fibers and Polymers: 2016; 17: 2078-2084.

[29] Yang T, Xiong X, Mishra R, Novak J and Militky J. Relationship Between Sound Absorption Property and Transmission Behavior of Struto Nonwoven. Paper presented at the Textile Bioengineering and Informatics Symposium Proceedings 2017 - 10th Textile Bioengineering and Informatics Symposium, TBIS 2017. 